\title{
Flash Continuous Glucose Monitoring and its IMPACT to REPLACE Blood Glucose Monitoring in the Management of Type 1 and Type 2 Diabetes
}

\author{
Stephen M Twigg, ${ }^{1}$ Mahmood R Kazemi, ${ }^{2}$ and Maria E Craig 3 \\ 1. Central Clinical School, Charles Perkins Centre, Bosch Institute, The University of Sydney, Australia; 2. Abbott Diabetes Care, Alameda, California, \\ US; 3. The Children's Hospital at Westmead, Westmead, and Charles Perkins Centre Westmead, The University of Sydney, Australia; School of \\ Women's and Children's Health, University of New South Wales, Sydney, Australia
}

DOI: https://doi.org/10.17925/USE.2017.13.02.57

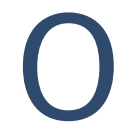

bjective: Established methods of self-monitoring of glucose levels include capillary self-monitoring of blood glucose (SMBG) and interstitial continuous glucose monitoring (CGM). Flash CGM is a novel form of self-monitoring that provides on-demand continuous interstitial glucose profiles. The purpose of this article is to critically review the recent outcome data from randomized controlled trials that assessed the efficacy and safety of flash CGM to replace routine SMBG in diabetes management. Methods: Two recent six-month, prospective, multicenter, randomized controlled trials in type 1 (IMPACT; NCT02232698) and type 2 (REPLACE; NCT02082184) diabetes compared flash CGM with SMBG under otherwise usual care conditions. The trials did not use a prescribed treatment algorithm based on self-monitoring of glucose. Results: Both trials demonstrated that the time spent in hypoglycemia over a 24-hour period, as well as overnight, was markedly reduced by flash CGM without deterioration in glycated hemoglobin (A1C) levels. In IMPACT there was a 38\% reduction in time in hypoglycemia with flash CGM versuS SMBG, and in REPLACE there was a 43\% reduction in time in hypoglycemia with flash CGM compared with SMBG. Moreover, patient satisfaction improved with flash CGM, usage adherence rates were high, and flash CGM was well tolerated. Conclusions: The findings from these trials suggest that improved care outcomes can be achieved when flash CGM is integrated into current established clinical care paradigms. Flash CGM provides important advantages over SMBG that are likely to be applicable to real-world care of individuals with differing forms of diabetes requiring intensive insulin treatment.

\section{Keywords}

Continuous glucose monitoring, diabetes, flash glucose monitoring, glycated hemoglobin, hypoglycemia, self-monitoring of blood glucose

Disclosure: Stephen M Twigg is an advisory panel member and is on the speaker's bureau for Abbott Diabetes Care, Astra Zeneca, Novo Nordisk Australia, Sanofi-Aventis, Eli Lilly and Company and Boehringer Ingelheim Pharmaceuticals. He is on the speaker's bureau only for Takeda Pharmaceutical Company Limited, Merck Sharp \& Dohme Corporation, Novartis Pharmaceuticals Corporation, and Servier. Mahmood R Kazemi is an employee of Abbott Diabetes Care. Maria E Craig is an advisory panel member for Abbott Diabetes Care, and is on the speaker's bureau for Novo Nordisk Australia, Pfizer Australia. Abbott Diabetes Care has contracted with Watermeadow Medical (UK) to manage and prepare this manuscript.

Acknowledgements: The authors thank Liz Southey and Rebecca Cocking of Watermeadow Medical (UK) for assistance with the preparation of this manuscript. Editoria support was provided by Catherine Amey, Touch Medical Media and funded by Abbott. Compliance with Ethics: This study involves a review of the literature and did not involve any studies with human or animal subjects performed by any of the authors.

Authorship: All named authors meet the International Committee of Medical Journal Editors (ICMJE) criteria for authorship of this manuscript, take responsibility for the integrity of the work as a whole, and have given final approval to the version to be published.

open Access: This article is published under the Creative Commons Attribution Noncommercial License, which permits any noncommercial use, distribution, adaptation, and reproduction provided the original author(s) and source are given appropriate credit.

Received: March 6, $2017 \quad$ Accepted: June 9, 2017

Citation: US Endocrinology, 2017;13(2):57-62

Corresponding Author: Stephen M Twigg, Sydney Medical School, Charles Perkins Centre (D17), Level 3 West, The University of Sydney, Sydney NSW 2006, Australia. E: stephen.twigg@sydney.edu.au

Support: The publication of this article was supported by Abbott, who was given the opportunity to review the article for scientific accuracy before submission. Any resulting changes were made at the authors' discretion.
The main priorities in diabetes care include prompt recognition and minimization of hypoglycemia while optimizing glucose control and glycated hemoglobin (A1C) levels. To achieve this, individuals need accurate and clear information about their glucose levels. There are practical limits to the frequency of self-monitoring of blood glucose (SMBG) that can be performed daily, which means that testing tends to focus on pre-meal glucose levels and relies on patient motivation and compliance. ${ }^{1}$ The intermittent snapshots of blood glucose provided by SMBG do not provide information for large parts of the day or night. Newer glucose monitoring approaches enable additional testing to be performed at regular intervals, and provide more information than would be feasible with SMBG. This includes comprehensive data on the 24-hour glucose profile; the current glucose trend (up or down); glucose variability; detection of periods of hypoglycemia and hyperglycemia; and estimated A1C.

\section{Advantages and disadvantages of currently available glucose monitoring approaches Self-monitoring of blood glucose}

SMBG based on finger-prick testing of capillary blood using glucose test strips is generally performed at intervals throughout the day (typically four to eight times daily, depending on the individual). ${ }^{2}$ Current guidelines for type 1 diabetes (T1D) recommend testing at a frequency to optimize diabetes control, because frequency of SMBG correlates with glycemic control. ${ }^{3}$ The practical limitations of SMBG mean that it has a limited frequency of use, and hence it does not provide optimal information to examine glucose variability, periods of hypoglycemic or hyperglycemic risk, 
Table 1: Key characteristics of different glucose monitoring methods*

\begin{tabular}{|c|c|c|c|}
\hline Parameter & SMBG & Traditional CGM & Flash CGM \\
\hline Fluid tested & Blood & Interstitial fluid & Interstitial fluid \\
\hline $\begin{array}{l}\text { User calibration } \\
\text { required }\end{array}$ & No & Twice daily & No \\
\hline $\begin{array}{l}\text { Maximum duration } \\
\text { of sensor use, days }\end{array}$ & Not applicable & 7 & 14 \\
\hline Number of tests & Limited & Virtually unlimited & Virtually unlimited \\
\hline $\begin{array}{l}\text { Report } \\
\text { interpretation }\end{array}$ & $\begin{array}{l}\text { Variable (hardware- } \\
\text { and software- } \\
\text { dependent) }\end{array}$ & $\begin{array}{l}\text { Can be complex } \\
\text { (software- } \\
\text { dependent) }\end{array}$ & $\begin{array}{l}\text { Relatively easy } \\
\text { (based on AGP) }\end{array}$ \\
\hline Operator training & Simple & Complex & Simple \\
\hline
\end{tabular}

$A G P=$ ambulatory g/ucose profile; $C G M=$ continuous g/ucose monitoring:

SMBG = self-monitoring of blood glucose. Sources: Kalra et al., 2015;" Heinemann et al., 2015. ${ }^{*}$ As per the text, this commentary critically reports upon recent outcomes of randomized controlled trials of flash continuous glucose monitoring versus self-monitoring of blood glucose.

or overnight glycemic control. Furthermore, finger-prick blood sampling can be painful. Along with cost, needle fear, and social stigma, poor motivation and inconvenience may contribute to variable adherence with SMBG. ${ }^{4}$ Direct financial costs associated with SMBG are lower than traditional continuous glucose monitoring (CGM) and possibly also flash continuous glucose monitoring (flash CGM), although the cost is dependent on the number of test strips used daily. ${ }^{2}$

\section{Traditional continuous glucose monitoring}

Traditional CGM is predominantly utilized by individuals with T1D who have received extensive training in how to use the monitoring system. It involves continuous glucose sensing with passive relay of information from sensor to receiver for use by the individual. ${ }^{2}$ Traditional CGM provides constantly updated information on glucose levels and can provide audible alarms if programmed glucose thresholds are at risk of being, or are, transgressed. CGM sensors can be linked to an insulin pump that automatically suspends insulin administration based on glucose readings. However, systems need to be calibrated twice daily with finger-prick capillary glucose measures. The cost of traditional CGM, which is significantly higher than SMBG in the $\mathrm{US}_{1}{ }^{2}$ may affect patient accessibility. Clinical adoption of traditional CGM is also hampered by a lack of standardized reporting of glucose data and difficulty in evaluating the large amount of data produced by such systems. ${ }^{5}$ Patient acceptability of, and therefore adherence to, traditional CGM use may limit the potential benefits of this monitoring approach. Studies have demonstrated that traditional CGM use declines over time, particularly in adolescents. ${ }^{6-8}$

\section{Flash continuous glucose monitoring}

Flash CGM systems comprise a reader and a factory-calibrated sensor (1.4 inches wide $\times 0.20$ inches high) with a filament inserted under the skin of the back of the arm and held in place by an adhesive. ${ }^{\text {Th }}$ The sensor is powered by a battery and functions for up to 14 days once activated. A 0.20 inch length filament on the sensor measures interstitial glucose at regular intervals and the sensor stores up to 8 hours of continuous glucose data. The sensor is scanned by a reader that provides a current glucose reading, glucose trend (indicated by an arrow), and an 8-hour continuous glucose profile. A key differentiating feature of flash CGM compared with traditional CGM is that flash CGM involves active user engagement to transfer data, which must be performed at least once every 8 hours to transfer all collected data. Data are stored in the reader for up to 90 days. This can be used to generate a detailed ambulatory glucose profile (AGP) report that facilitates detection of glycemic patterns and trends over the duration of sensor wear. Flash CGM does not require user calibration and replaces routine SMBG for treatment decisions, except in the following circumstances: if hypoglycemia or impending hypoglycemia is reported, when clinical symptoms do not match the system readings, or during periods of rapid glucose change. ${ }^{2}$

Flash CGM provides individuals with easily accessible information on their glucose levels over the whole day, identifies times of day when they are more at risk of low or high glucose levels, and details their glucose variability. This information can enable individuals to optimize their glycemic control, while avoiding undue hypoglycemic risk. The flash CGM system does not produce audible alarms when glucose levels are beyond specified thresholds, which may be beneficial in reducing alarm fatigue, a common cause for discontinuation of traditional CGM devices. This can occur when users experience too many alarms overall and false alarms in particular, which may lead them to either ignore or silence hypoglycemia alarms. ${ }^{9}$ However, for other individuals who find alarms helpful, the lack of this feature may be a limitation. In addition, given that flash CGM is a relatively new method, there are fewer studies available than with SMBG and traditional CGM. Consequently, currently flash CGM is not widely mentioned in established clinical care guidelines. The cost of flash CGM is lower than that of traditional CGM, and is potentially similar to SMBG depending on the number of blood glucose test strips used.?

There are a number of differences across the various glucose monitoring methods (see Table 1).1,2 In particular, SMBG measures capillary blood glucose, whereas both traditional CGM and flash CGM measure interstitial glucose. Flash CGM measurements have been shown to be clinically accurate compared with capillary measurements over 14 days of wear, and are unaffected by patient factors such as body mass index, age, type of diabetes, insulin use, or A1C level. ${ }^{10}$ Furthermore, regular glucose testing with flash CGM or traditional CGM provides a more informative glucose profile versus SMBG. Flash CGM report interpretation is aided by both realtime trend profiles and standardized AGP software. ${ }^{11}$ This software presents data over a representative 24-hour period and provides glucose pattern insights. A distinguishing feature of current flash CGM systems is the lack of need for user calibration, which may add to the convenience of flash CGM over traditional CGM systems.

Recently, two large clinical trials, conducted with flash CGM in individuals with T1D and type 2 diabetes (T2D), have been published. .2,13 $^{12}$ The objective of this review is to summarize recent data from these randomized controlled trials which assessed the efficacy and safety of flash CGM to replace routine SMBG in diabetes management.

\section{Recent clinical trials of flash continuous glucose monitoring in type 1 diabetes and type 2 diabetes Study designs}

IMPACT (ClinicalTrials.gov NCT02232698) was a prospective, randomized, controlled, two-arm study conducted at 23 European diabetes centers in Sweden, Austria, Germany, Spain, and the Netherlands.12 REPLACE (NCT02082184) was a prospective, randomized, controlled, two-arm study involving 26 European centers in France, Germany, and the UK..$^{13}$ These trials 
Figure 1: IMPACT (A) and REPLACE (B) study designs

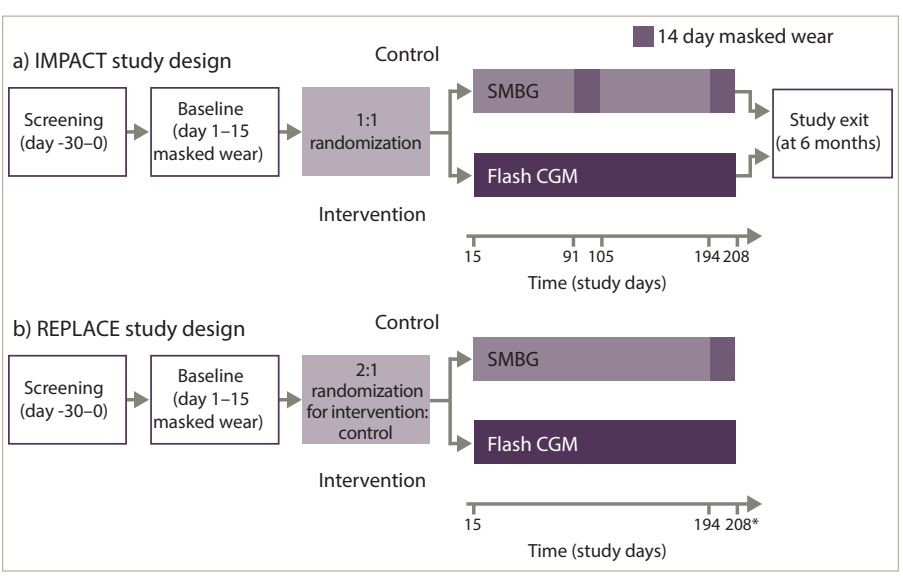

Flash CGM = flash continuous glucose monitoring; SMBG = self-monitoring of blood glucose. IMPACT: control individuals wore the masked sensor at 3 and 6 months. REPLACE: individuals were randomized in a 2:1 ratio to flash CGM and SMBG; individuals wore the masked sensor at 6 months. *An open access phase (additional 180 days) followed the study period in REPLACE, but it was not part of the primary endpoint nor reported in the primary manuscript. Reproduced with permissions from: Bolinder J et al., 2016. ${ }^{12}$

examined the use of the FreeStyle Libre ${ }^{\text {TM }}$ (Abbott Diabetes Care, Alameda, California, US) flash CGM system compared with SMBG in the management of T1D and T2D, respectively.

IMPACT randomized 241 adults aged $\geq 18$ years with T1D for $\geq 5$ years, who had been on their current insulin regimen for $\geq 3$ months prior to study entry, had a screening for $\mathrm{A} 1 \mathrm{C} \leq 7.5 \%(58 \mathrm{mmol} / \mathrm{mol})$, and had reported blood glucose readings on a regular basis equivalent to $\geq 3$-times daily for $\geq 2$ months prior to study entry. ${ }^{12}$ REPLACE randomized 224 adults aged $\geq 18$ years with T2D treated with intensive insulin therapy, A1C 7.5-12.0\% (58-108 $\mathrm{mmol} / \mathrm{mol}$ ) and $\geq 10$ blood glucose tests/week for $\geq 2$ months prior to study entry. ${ }^{13}$ For safety reasons, individuals judged to have ongoing, clinically significant hypoglycemia unawareness were excluded from either study.

IMPACT and REPLACE shared a similar study design (see Figure 1). During baseline, all individuals wore a masked sensor system (modified Freestyle LibreT⿳) for 14 days. Throughout this period, sensor glucose measurements were blinded for both the participant and the investigator. Diabetes management during this period was performed based on SMBG. Individuals were randomized 1:1 (IMPACT) and 2:1 (REPLACE) to utilizing flash CGM sensor data for self-management or continuing to use SMBG. There were no protocol-mandated insulin adjustments; instead, individuals adjusted their treatment based on local clinical care protocols. Individuals were encouraged to self-manage utilizing current or historical glucose data including avoidance of hypoglycemia. Glucose control and readings in both groups were formally reviewed at clinic visits. ${ }^{12}$ In T2D, management aimed to avoid hypoglycemia, optimize fasting glucose, and reduce postprandial glucose excursions..$^{13}$ At 3 and 6 months in IMPACT, and at 6 months in REPLACE, individuals in the control group wore masked sensors for reassessment of glycemic parameters. A key limitation of the study design of IMPACT was that patients with quite well-controlled T1D (with A1C below 7.5\% [59 mmol/mol]) were included since such patients are theoretically at higher risk of hypoglycemia ${ }^{14}$ and hypoglycemia duration was the primary endpoint of the study. Recruited participants may not be representative of the eligible population of individuals with
Table 2: Clinical endpoints in the IMPACT and REPLACE trials

\begin{tabular}{|c|c|c|}
\hline Endpoints & IMPACT & REPLACE \\
\hline Primary & $\begin{array}{l}\text { Time in hypoglycemia }(<70 \mathrm{mg} / \mathrm{dL} \\
[<3.9 \mathrm{mmol} / \mathrm{L}])\end{array}$ & A1C level \\
\hline $\begin{array}{l}\text { Pre- } \\
\text { specified } \\
\text { key } \\
\text { secondary }\end{array}$ & $\begin{array}{l}\text { Change in A1C level } \\
\text { Number and duration of daytime and } \\
\text { nocturnal hypoglycemic episodes } \\
\text { Time in range } 70-180 \mathrm{mg} / \mathrm{dL} \\
\text { (3.9-10.0 mmol/L) } \\
\text { Time in hyperglycemia } \\
\text { Glucose variability measurements } \\
\text { Blood glucose testing and sensor } \\
\text { scan frequency }\end{array}$ & $\begin{array}{l}\text { Number and duration of } \\
\text { hypoglycemic episodes } \\
\text { Time in range } 70-180 \mathrm{mg} / \mathrm{dL} \\
\text { [3.9-10.0 mmol/L] } \\
\text { Time in hyperglycemia } \\
\text { Glucose variability } \\
\text { measurements } \\
\text { Blood glucose testing and } \\
\text { sensor scan frequency }\end{array}$ \\
\hline
\end{tabular}

T1D. Similarly, in REPLACE, only patients receiving intensive insulin therapy performing regular glucose testing were included, again limiting the generalizability of the data. For example, people with T2D receiving basal insulin injections only or biphasic insulin were not included in the REPLACE study.

\section{Primary endpoints}

The primary study endpoints were the difference in time in hypoglycemia $<70 \mathrm{mg} / \mathrm{dL}(<3.9 \mathrm{mmol} / \mathrm{L})$ in IMPACT, and the difference in A1C in REPLACE (see Table 2). ${ }^{12,13}$

\section{Key secondary endpoints}

The key secondary endpoints in IMPACT and REPLACE are presented in Table 2. They included time in range (70-180 mg/dL [3.9-10.0 mmol/L]), time in hyperglycemia, and glucose variability. ${ }^{12,13}$

\section{Safety}

Adverse events (AEs) and sensor insertion-site symptoms were monitored throughout the study. In addition, severe hypoglycemia (requiring thirdparty assistance) was assessed.

\section{Clinical implications of flash continuous glucose monitoring}

The study designs of IMPACT and REPLACE reflect real-world clinical practice, as there were no protocol-mandated treatment adjustments. 12,13 As such, individual centers utilized flash CGM to inform and guide treatment based on local clinical practice. Flash CGM was found to result in significant reductions in hypoglycemia in both trials, regardless of the threshold or type of hypoglycemia measure. In IMPACT (T1D), the daily time in hypoglycemia was reduced by $1.4 \mathrm{~h}$ /day in the flash CGM group (from 3.4 to $2.0 \mathrm{~h} /$ day) and by $0.2 \mathrm{~h}$ (from 3.4 to $3.3 \mathrm{~h} /$ day) in the SMBG group (mean between-group difference \pm standard error [SE]: $-1.2 \pm 0.2 \mathrm{~h} /$ day; $\mathrm{p}<0.0001$ ). This equates to a $38 \%$ reduction in time in hypoglycemia with flash CGM versus SMBG (see Figure 2A). In REPLACE (T2D), daily time in hypoglycemia was reduced by $0.7 \mathrm{~h} /$ day (from 1.3 to $0.6 \mathrm{~h} /$ day) in the flash CGM group and by $0.1 \mathrm{~h} /$ day (from 1.1 to $1.0 \mathrm{~h} /$ day) in the SMBG group (mean betweengroup difference $\pm \mathrm{SE}:-0.5 \pm 0.1 \mathrm{~h} / \mathrm{day} ; \mathrm{p}=0.0006$ ). This equates to a $43 \%$ reduction in time in hypoglycemia with flash CGM, compared with SMBG (see Figure 2B). In IMPACT, there were seven hypoglycemia-related serious AES (requiring hospitalization or third-party intervention), occurring in six individuals: two in the intervention group (two individuals) and four in the control group (three individuals). 
Figure 2: Time in glycemic range with SMBG (control) and flash CGM (intervention) in IMPACT (A) and REPLACE (B)

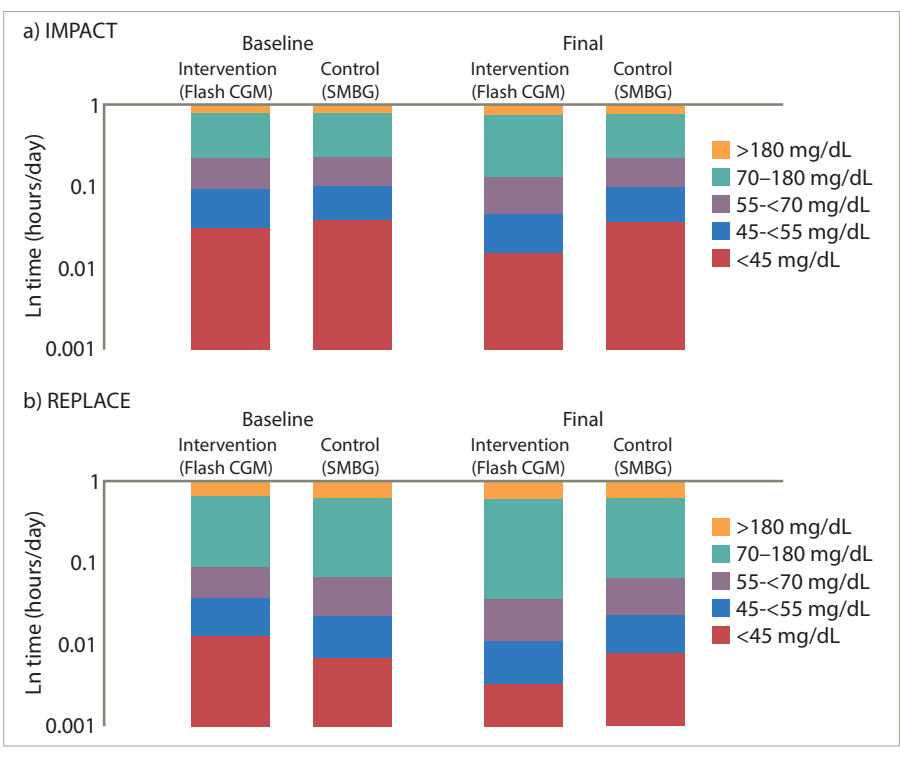

Flash CGM = flash continuous glucose monitoring; $L n=$ logarithmic scale; $S M B G=$ selfmonitoring of blood glucose. Hypoglycemia $(<55$ and $<70 \mathrm{mg} / \mathrm{dL}$ ) was reduced $(p<0.0001$ and $p<0.0001$, respectively) in each clinical trial by the flash continuous glucose monitoring intervention.

Among the secondary endpoints, IMPACT and REPLACE also demonstrated significant reductions in time in nocturnal hypoglycemia (occurring between 23:00 and 06:00) with flash CGM, notably in the absence of alarms. In T1D, time in nocturnal hypoglycemia $70 \mathrm{mg} / \mathrm{dL}(<3.9 \mathrm{mmol} / \mathrm{L})$ was reduced by $40 \%$ with flash CGM compared with control ( $p<0.0001)$. Time in nocturnal hypoglycemia was reduced by $0.64 \mathrm{~h}$ with flash CGM (1.32 h to $0.68 \mathrm{~h})$ and by $0.25 \mathrm{~h}(1.48 \mathrm{~h}$ to $1.23 \mathrm{~h}$ ) with SMBG (mean between-group difference: $-0.47 \mathrm{~h}$ ). In T2D, time in nocturnal hypoglycemia $70 \mathrm{mg} / \mathrm{dL}$ (<3.9 mmol/L) was reduced by $54 \%$ with flash CGM versus control $(p=0.0001)$. Time in nocturnal hypoglycemia decreased from 0.55 to $0.23 \mathrm{~h}$ with flash CGM, and was unchanged from 0.49 to $0.51 \mathrm{~h}$ with SMBG (mean between-group difference: -0.29 h).

Despite a reduction in hypoglycemia with flash CGM in both T1D and T2D, A1C did not increase in either study. ${ }^{12,13}$ In T1D, there was no significant adjusted mean change in A1C from baseline with flash CGM (adjusted mean difference in A1C between flash CGM and SMBG \pm SE: $0.00 \pm$ $0.06 \% ; \mathrm{p}=0.96) .{ }^{12}$ In T2D, a reduction in A1C was reported in the flash CGM group $(-0.29 \pm 0.07 \%)$ and in the control group $(-0.31 \pm 0.09 \%$; adjusted mean difference in A1C between flash CGM and SMBG \pm SE: $0.03 \pm$ $0.11 \% ; \mathrm{p}=0.82){ }^{13}$ Therefore, individuals in IMPACT and REPLACE made appropriate insulin dosing decisions based on sensor readings, with less hypoglycemia experienced than control individuals who utilized SMBG for these decisions. 10,12,13 Glycemic clinical outcomes in both IMPACT and REPLACE were positive, in that there was no compromise in A1C with flash CGM versus SMBG in either study, despite the reduction in hypoglycemia with flash CGM. ${ }^{12,13}$ With respect to glucose variability, in IMPACT, time in the target glucose range was improved with flash CGM compared with SMBG by $1.0 \pm 0.30 \mathrm{~h} /$ day (adjusted mean $\pm S E ; p=0.0006)^{12}$; glucose variability was improved with flash CGM versus SMBG in both studies (glucose coefficient of variation: difference in IMPACT $-4.4 \pm 0.6, p<0.0001$; difference in REPLACE $-2.3 \pm 0.7, \mathrm{p}=0.0017)$.
Table 3: Key quality of life outcomes from IMPACT and REPLACE

\begin{tabular}{|l|l|l|l|}
\hline Endpoint & $\begin{array}{l}\text { Flash CGM } \\
\text { adjusted mean }\end{array}$ & $\begin{array}{l}\text { SMBG adjusted } \\
\text { mean }\end{array}$ & p-value \\
\hline IMPACT & 13.3 & 7.2 & $<0.0001$ \\
\hline $\begin{array}{l}\text { DTSQ total treatment satisfaction } \\
\text { (change score) }\end{array}$ & -0.17 & 0.07 & $<0.0001$ \\
\hline $\begin{array}{l}\text { Change from baseline DQOL } \\
\text { satisfaction with treatment }\end{array}$ & 13.1 & 9.0 & $<0.0001$ \\
\hline \begin{tabular}{l} 
REPLACE \\
\hline $\begin{array}{l}\text { DTSQ total treatment satisfaction } \\
\text { (change score) }\end{array}$
\end{tabular} & -0.2 & 0.0 & 0.0259 \\
\hline $\begin{array}{l}\text { Change from baseline DQOL } \\
\text { satisfaction with treatment }\end{array}$ & & & \\
\hline
\end{tabular}

DQOL = Diabetes Quality of Life; DTSQ = Diabetes Treatment Satisfaction Questionnaire; Flash CGM = flash continuous g/ucose monitoring: $S M B G=$ self-monitoring of blood glucose.

In both studies, blood glucose testing was significantly reduced in the flash CGM group and was effectively replaced with sensor readings. In the flash CGM group in IMPACT, the mean number of sensor scans was 15.1 per day, and the mean number of SMBG tests decreased from 5.5 per day at baseline to 0.5 per day at 6 months; in the SMBG group, the number of SMBG tests remained constant (5.8 per day at baseline; 5.6 per day at 6 months). ${ }^{12}$ Similarly, in the flash CGM group in REPLACE, the mean number of sensor scans was 8.3 per day, and the mean number of SMBG tests fell from 3.8 per day at baseline to 0.3 per day over 6 months; in the SMBG group, there was no change in the number of SMBG tests (3.9 per day at baseline and 3.8 at 6 months). ${ }^{13}$

Based on computer modeling (in silico simulations), it has been suggested that glucose sensors with a mean absolute relative difference (MARD) of $10 \%$ or less may be considered safe for insulin dosing decisions. ${ }^{15}$ However, in IMPACT and REPLACE, real-world use of a sensor system with a published MARD of $11.4 \%{ }^{10}$ resulted in appropriate insulin dosing decisions, significantly less hypoglycemia, and comparable glycemic control versus SMBG. Therefore, clinical data from IMPACT and REPLACE do not support an in silico-defined MARD threshold of $\leq 10 \%$ for sensor-based treatment decisions in adults. These data are likely of greater value to the clinician as they reflect real-world clinical use of flash CGM rather than in silico simulations of sensor use. 12,13,15

Quality of life (QOL) data in both IMPACT and REPLACE showed greater treatment satisfaction with flash CGM compared with SMBG. In IMPACT, individuals with T1D reported significantly improved results in the Diabetes Treatment Satisfaction Questionnaire (DTSQ) total treatment satisfaction with flash CGM versus SMBG (13.3 and 7.2, respectively; $p<0.0001$ ) (see Table 3). Similarly, Diabetes Quality of Life (DQoL) satisfaction with treatment, which evaluates the burden of an intensive diabetes treatment regimen, was significantly improved with flash CGM versus SMBG $(-0.17$ and 0.07 , respectively; $\mathrm{p}<0.0001) .{ }^{13}$ In REPLACE, individuals with T2D utilizing flash CGM had a significantly improved mean DTSQ total treatment satisfaction score of 13.1 versus 9.0 with SMBG $(p<0.0001)$. DQoL satisfaction with treatment was also significantly improved with flash CGM compared with SMBG $(-0.2$ versus 0.0 , respectively; $\mathrm{p}=0.0259$ ). These data indicate improved QoL in individuals with either type of diabetes using insulin therapy. 
Adherence to SMBG has been reported to be as low as 44\% in T1D and $24 \%$ in T2D. ${ }^{16}$ Given that flash CGM provides continuous, unobtrusive, and convenient glucose monitoring without the need for routine blood glucose tests, it may improve patient adherence versus SMBG. High rates of adherence to flash CGM were seen in both studies: the percentage of available data collected was $92.8 \%$ in IMPACT and $88.7 \%$ in REPLACE. This high rate of adherence to flash CGM was associated with >94\% $(n=68 / 72)$ of individuals giving favorable ratings for flash CGM compared to fingerstick testing, in terms of sensor comfort, ease of wear, compatibility with everyday activities, pain experienced, ease of use, and erythema or edema occurring when the sensor was removed..$^{10}$

Flash CGM provides users with detailed, readily accessible glucose data. The flash CGM reader provides a continuous glucose profile over the previous 8 hours and the glucose trend, as well as historical data over the previous 90 days (see Figure $3 A$ ). Data provided by the sensor can be analyzed to generate a standardized AGP report, which facilitates glucose pattern recognition over a representative 24-hour period (see Figure 3B). ${ }^{11}$ The AGP provides users with information on hypoglycemia and hyperglycemia trends, and can quickly identify times when glucose control is not optimal. AGP gives both individuals with diabetes and their healthcare providers (HCPS) an important tool for understanding their glycemic patterns and can be used by HCPs for educational purposes. ${ }^{1}$

Flash CGM was well tolerated in both studies. Common AEs in the two trials included skin reactions related to wearing the sensor. ${ }^{12,13}$ In IMPACT, 13 sensor site reactions were reported in 10/119 individuals (8.4\%). In REPLACE, 6/149 individuals (4.0\%) experienced sensor site reaction AES. The event rate of these reactions was well within what would be expected for a device with a medical-grade adhesive. In IMPACT, 215 anticipated sensor insertion-site symptoms (non-adverse events) were reported in 47/119 individuals (39.5\%). In REPLACE, 143 anticipated sensor insertionsite symptoms were reported in 41/149 individuals (27.5\%).

In both T1D (IMPACT) and T2D (REPLACE), flash CGM allowed users to significantly decrease their time in hypoglycemia without worsening glycemic control as measured by A1C. These benefits were accompanied by significant decreases in blood glucose testing and effective replacement of sensor readings for SMBG in treatment decisions. The clinical benefits observed may reflect improved management by individuals empowered by the comprehensive and convenient glucose measurements available with flash CGM, as well as the more easily accessible historical data. For example, the reductions in nocturnal hypoglycemia without alarms may reflect individuals' ability to utilize historical sensor glucose data trends and patterns to make therapy changes, perhaps by adjusting their bedtime insulin dose or pre-bedtime carbohydrate intake. The observation that reductions in hypoglycemia occurred within the first two weeks of real-time sensor use provides further evidence that patientdriven changes in therapy based on access to comprehensive glucose information from flash CGM may underlie the clinical benefits seen with this new technology.

\section{Conclusions}

The clinical trial experience with flash CGM in T1D (IMPACT) and T2D (REPLACE) demonstrates statistically and clinically significant benefits in
Figure 3: Flash continuous glucose monitoring provides readily accessible and insightful glucose monitoring profiles

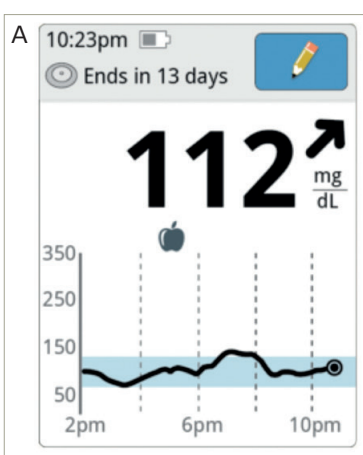

B Glucose Pattern Insights

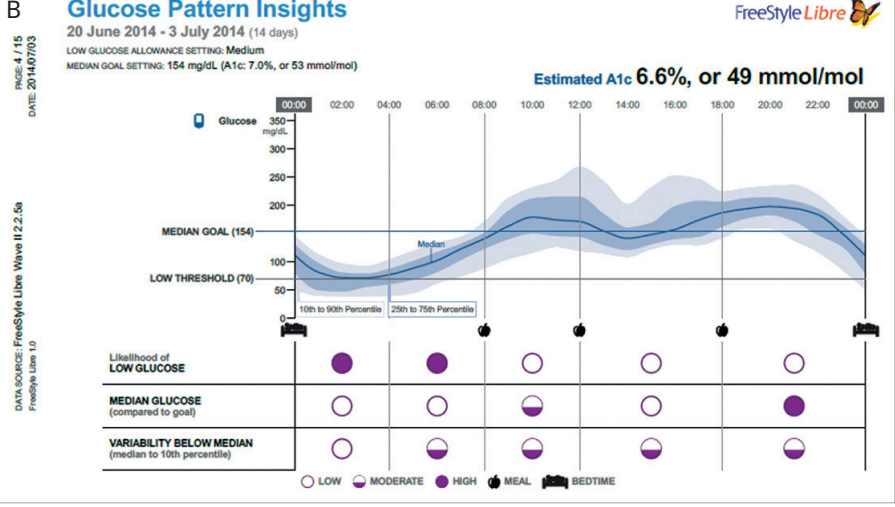

$A G P=$ ambulatory glucose profile; Flash CGM = flash continuous glucose monitoring. Reader display with continuous glucose data provided over the previous 8 hours, as well as the current glucose reading and a trend arrow (A). Example of an ambulatory glucose report, including 14 days of data derived from the Freestyle Libre TM flash CGM System (B). The user-friendly report helps to identify when desirable outcomes in glycemic control are being realized (empty circles) and areas for concern and improvement (partially or completely filled circles). In this example, the main issues of concern identified are low glucose readings overnight and into the early morning hours, and moderate glucose variability between the days of data collection (B).

the reduction of nocturnal and overall hypoglycemia without compromising glycemic control. These findings have implications for improving the care of individuals with diabetes on insulin therapy. Reducing the occurrence of hypoglycemia has important effects on patient QoL, including benefits in both short- and long-term physical wellbeing, ability to maintain work and life commitments, and self-confidence in disease management. Enabling individuals to manage their glucose levels while minimizing hypoglycemia facilitates increased confidence to achieve better control. ${ }^{17}$ Interestingly, in both IMPACT and REPLACE, individuals reported improved QOL and greater treatment satisfaction with flash CGM versus SMBG. ${ }^{12,13}$ Previous studies have reported perceived diabetes control as a key aspect of increasing treatment satisfaction. ${ }^{18}$ It is possible that the improvements in QoL in IMPACT and REPLACE at least partly reflect a perception among individuals in the flash CGM group that they were more in control of their diabetes. Flash CGM is still relatively new but these key clinical trials in T1D (IMPACT) and T2D (REPLACE) suggest that improved outcomes can be achieved when this technology is integrated into established clinical care paradigms. Further comparative data are needed, in particular, from head-to-head studies comparing flash CGM with traditional CGM. Such studies would provide a better understanding of the relative advantages of flash CGM and its role in diabetes management. $\square$ 


\section{Review Diabetes}

1. Kalra S, Gupta Y, Ambulatory glucose profile: Flash glucose monitoring, J Pak Med Assoc, 2015;65:1360-2

2. Heinemann L, Freckmann G, CGM Versus FGM; or, Continuous Glucose Monitoring Is Not Flash Glucose Monitoring I Diabetes Sci Technol, 2015;9:947-50.

3. Rewers MJ, Pillay K de Beaufort C, et al., ISPAD Clinical Practice Consensus Guidelines 2014. Assessment and monitoring of glycemic control in children and adolescents with diabetes, Pediatr Diabetes, 2014;15(Suppl 20):102-14.

4. Ong WM, Chua SS, Ng CJ, Barriers and facilitators to selfmonitoring of blood glucose in people with type 2 diabetes using insulin: a qualitative study, Patient Prefer Adherence, 2014;8:237-46.

5. Kohnert KD, Heinke P, Vogt L, Salzsieder E, Utility of different glycemic control metrics for optimizing management of diabetes, World I Diabetes, 2015;6:17-29.

6. Slover RH, Welsh JB, Criego A, et al., Effectiveness of sensoraugmented pump therapy in children and adolescents with type 1 diabetes in the STAR 3 study, Pediatr Diabetes, 2012;13:6-11.

7. Tanenberg RJ, Welsh JB, Patient behaviors associated with optimum glycemic outcomes with sensor-augmented pump therapy: insights from the star 3 study, Endocr Pract, 2015:21:41-5
8. Juvenile Diabetes Research Foundation Continuous Glucose Monitoring Study Group, Effectiveness of continuous glucose monitoring in a clinical care environment: evidence from the Juvenile Diabetes Research Foundation continuous glucose monitoring (JDRF-CGM) trial, Diabetes Care, 2010:33:17-22.

9. Shivers JP. Mackowiak L, Anhalt H, Zisser H, "Turn it off!": diabetes device alarm fatigue considerations for the present and the future, J Diabetes Sci Technol, 2013;7:789-94.

10. Bailey T, Bode BW, Christiansen MP, The Performance and Usability of a Factory-Calibrated Flash Glucose Monitoring System, Diabetes Technol Ther, 2015;17:787-94.

11. Bergenstal RM, Ahmann AJ, Bailey T, et al., Recommendations for standardizing glucose reporting and analysis to optimize clinical decision making in diabetes: the ambulatory glucose profile, J Diabetes Sci Technol, 2013;7:562-78.

12. Bolinder J, Antuna R, Geelhoed-Duijvestijn P, et al., Novel glucosesensing technology and hypoglycaemia in type 1 diabetes: a multicentre, non-masked, randomised controlled trial, Lancet, 2016;388:2254-63.

13. Haak T, Hanaire $H$, Ajjan R, et al., Flash Glucose-Sensing Technology as a Replacement for Blood Glucose Monitoring for the Management of Insulin-Treated Type 2 Diabetes: a
Multicenter, Open-Label Randomized Controlled Trial, Diabetes Ther, 2017;8:55-73

14. UK Prospective Diabetes Study (UKPDS) Group, Intensive bloodglucose control with sulphonylureas or insulin compared with conventional treatment and risk of complications in patients with type 2 diabetes (UKPDS 33), Lancet, 1998:352:837-53.

15. Kovatchev BP, Patek SD, Ortiz EA, Breton MD, Assessing sensor accuracy for non-adjunct use of continuous glucose monitoring, Diabetes Technol Ther, 2015;17:177-86.

16. Patton SR, Adherence to glycemic monitoring in diabetes, J Diabetes Sci Technol, 2015;9:668-75.

17. Ahren B, Avoiding hypoglycemia: a key to success for glucoselowering therapy in type 2 diabetes, Vasc Health Risk Manag, 2013:9:155-63.

18. Polonsky W, Traylor L, Wei W, et al., More satisfied, but why? A pooled patient-level analysis of treatment satisfaction following the initiation of insulin glargine vs. comparators in insulin-naive patients with type 2 diabetes mellitus, Diabetes Obes Metab, 2014;16:255-61.

19. Kovatchev BP, Clarke WL, Breton M, et al., Quantifying temporal glucose variability in diabetes via continuous glucose monitoring mathematical methods and clinical application, Diabetes Technol Ther $2005: 7: 849-62$ 